\title{
Continuous venovenous hemofiltration versus continuous venovenous hemodiafiltration in critically ill patients: A retrospective cohort study from a Canadian tertiary centre
}

\author{
Farhan AlEnezi MD¹, Waleed Alhazzani MD MSc FRCPC ${ }^{1,2}$, Jinhui Ma PhD³, Safug Alanazi MD², Mary Salib MD, \\ Menat Attia $\mathrm{MD}^{4}$, Lehana Thabane $\mathrm{PhD}^{1,3,5}$, Alison Fox-Robichaud MD MSc FRCPC ${ }^{2}$
}

\begin{abstract}
F AlEnezi, W Alhazzani, J Ma, et al. Continuous venovenous hemofiltration versus continuous venovenous hemodiafiltration in critically ill patients: A retrospective cohort study from a Canadian tertiary centre. Can Respir J 2014;21(3):176-180.
\end{abstract}

BACKGROUND: Studies comparing continuous renal replacement therapy modalities are lacking. Theoretically, continuous venovenous hemofiltration $(\mathrm{CVVH})$ could be more effective than continuous venovenous hemodiafiltration (CVVHDF), and may be associated with fewer complications; however, there are no published data to support this hypothesis.

OBJECTIVE: To examine the effect of CVVH on mortality and other clinically important outcomes compared with CVVHDF in the intensive care unit (ICU) setting.

METHODS: Using a log of all continuous renal replacement therapy performed at a Canadian tertiary centre between 2007 and 2010, the records of patients meeting the inclusion criteria of being admitted to the ICU, and receiving either CVVH or CVVHDF for management of acute renal failure, were reviewed. The information retrieved included demographic data, death events, and hospital and ICU length of stay.

RESULTS: Data from 153 patients were included in the present study. Hospital and 30-day mortality were similar in the CVVH and CVVHDF groups (OR 0.85 [95\% CI 0.38 to 1.89]; $\mathrm{P}=0.69$ and $\mathrm{OR} 1.35$ [95\% CI 0.62 to 2.95]; $\mathrm{P}=0.45$, respectively). There was no difference in hospital length of stay (mean difference -34.14 [95\% CI -72.92 to 4.65]; $\mathrm{P}=0.08$ ). CONCLUSION: The present retrospective review suggests that the use of CVVH does not reduce mortality or hospital length of stay when compared with CVVHDF. Future randomized trials should control for different patient populations and continue to evaluate the removal of small molecules such as hormones.

Key Words: Acute renal failure; Cohort study; Continuous renal replacement therapy; Critically ill patients

\section{L'hémofiltration veino-veineuse continue ou}

l'hémodiafiltration veino-veineuse continue chez des patients gravement malades : une étude rétrospective de cohorte dans un centre canadien de soins tertiaires

HISTORIQUE : Il n'existe pas d'études comparant les modalités de thérapie continue en remplacement rénal. En théorie, l'hémofiltration veinoveineuse continue (HVVC) pourrait être plus efficace que l'hémodiafiltration veino-veineuse continue (HDFVVC) et provoquerait moins de complications. Cependant, il n'y a pas de données publiées pour soutenir cette hypothèse.

OBJECTIF : Examiner l'effet de l'HVVC sur la mortalité et d'autres résultats importants sur le plan clinique par rapport à l'HDFVVC à l'unité de soins intensifs (USI).

MÉTHODOLOGIE : Au moyen d'un journal de toutes les thérapies continues en remplacement rénal effectuées dans un centre canadien de soins tertiaires entre 2007 et 2010, les chercheurs ont examiné le dossier des patients respectant les critères d'inclusion, soit l'hospitalisation à l'USI l'administation d'une HVVC ou d'une HDFVVC pour traiter l'insuffisance rénale aiguë. Ils ont extrait les données démographiques, les décès et la durée du séjour à l'hôpital et à l'USI.

RÉSULTATS : Les données de 153 patients ont fait partie de la présente étude. L'hospitalisation et le taux de décès au bout de 30 jours étaient similaires dans les groupes d'HVVC et d'HDFVVC (RC 0,85 [95 \% IC 0,38 à 1,89]; $\mathrm{P}=0,69$ et RC 1,35 [95 \% IC 0,62 à 2,95]; $\mathrm{P}=0,45$, respectivement). Il n'y avait pas de différence dans la durée d'hospitalisation (différence moyenne $-34,14$ [95 \% IC -72,92 à 4,65]; $\mathrm{P}=0,08$ ).

CONCLUSION : D'après la présente analyse rétrospective, l'HVVC ne réduit pas le taux de mortalité ou la durée d'hospitalisation davantage que l'HDFVVC. Dans de prochains essais, il faudrait contrôler diverses populations de patients et continuer d'évaluer la suppression de petites molécules comme les hormones.

diffusion and convection using a highly efficient hemodiafilter to remove both solute and fluid. In continuous venovenous hemofiltration $(\mathrm{CVVH})$, a large ultrafiltrate volume is generated across a high-permeability membrane (convection). Convective modalities (hemofiltration) may provide enhanced clearance of middle molecular weight solutes compared with diffusive modalities (hemodialysis) $(15,16)$.

There is variation in practice worldwide. In Europe, clinicians primarily use $\mathrm{CVVH}$; in a recent survey from the United Kingdom, $61 \%$ of intensive care units (ICUs) who completed the survey used CVVH as a first-line therapy (17). In North America, both CVVH and CVVHDF are used (18), while in Australia and New Zealand, CVVHDF is used more frequently (19). A recent prospective observational study involving 54 ICUs from 23 countries (20) suggested that CVVH is more commonly used than CVVHDF (53\% versus $34 \%$, respectively).

It is unknown whether the effects of using CVVH differ from CVVHDF with regard to important patient outcomes. The primary Continuous venovenous hemodiafiltration (CVVHDF) combines

Departments of ${ }^{1}$ Anesthesia; ${ }^{2}$ Medicine; ${ }^{3}$ Clinical Epidemiology and Biostatistics; ${ }^{4}$ Michael DeGroote School of Medicine, McMaster University

${ }^{5}$ Biostatistics Unit, St Joseph's Healthcare, Hamilton, Ontario

Correspondence: Dr Alison Fox-Robichaud, Department of Medicine, McMaster University, DBR1 C5-106, 237 Barton Street East, Hamilton,

Ontario L8L 2X2. Telephone 905-521-2100 ext 40742, fax 905-575-2646, e-mail afoxrob@mcmaster.ca 


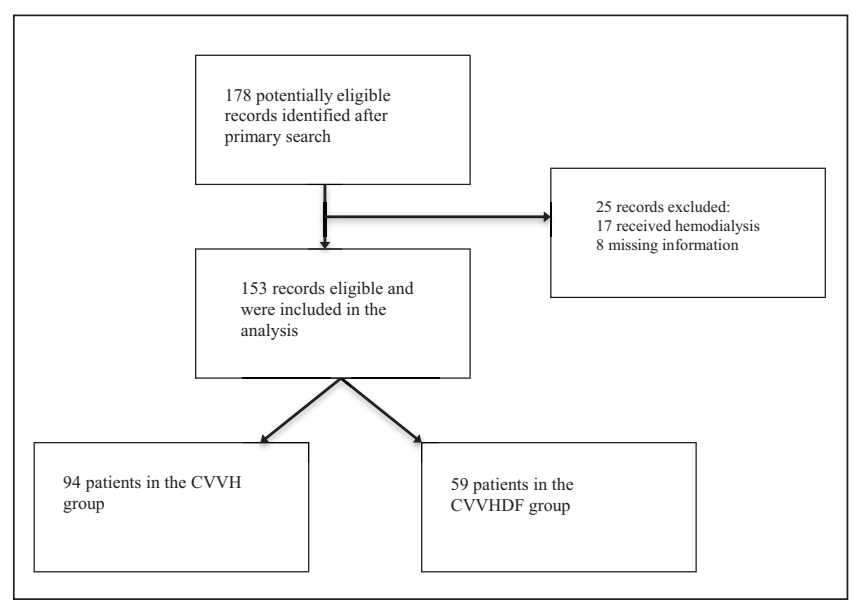

Figure 1) Flow diagram. CVVH Continuous venovenous hemofiltration; CVVHDF Continuous venovenous hemodiafiltration

purpose of the present study was to examine the effect of CVVH compared with CVVHDF on hospital mortality, 30-day mortality, ICU length of stay and hospital length of stay among critically ill patients with AKI. The secondary aims were to assess the effect of CVVH compared with CVVHDF on lactate and creatinine clearance, thyroid replacement and glucocorticoid levels in critically ill patients with AKI. For subgroup analysis in the postoperative cardiac population, we hypothesized that postcardiac surgery patients would have more benefit with convective therapy using CVVH because these patients carry a high risk of death in the context of AKI requiring dialysis postoperatively (21).

\section{Study design}

\section{METHODS}

A retrospective cohort study involving patients admitted to three ICUs (43 beds) in one Canadian tertiary care centre (Hamilton General Hospital [Hamilton, Ontario]) from 2007 to 2010 was performed. The study received research ethics approval from the Hamilton Health Sciences Research Ethics Board.

\section{Study population}

The inclusion criteria were as follows: age $\geq 18$ years; admission to the ICU; primary mode of dialysis received was either CVVH or CVVHDF for AKI requiring renal replacement therapy. CVVH was the primary mode used for CRRT at the authors' institution until 2008, when CVVHDF was introduced and became the primary CRRT mode. Patients who received intermittent hemodialysis as the initial dialysis mode and records with missing information were excluded (Figure 1). In the authors' centre, ST150 membrane filters are used on a Prismaflex (Gambro, USA) with a 13.5 Fr dialysis catheter; the replacement fluid is administered post filter. Using a predesigned hospital protocol, heparin is administered to prevent clotting of the filter. The majority of the dose is administered as CVVHD.

\section{Data collection}

Records for patients admitted to the ICU between 2007 and 2010 were reviewed through a log maintained by the ICU educators. Four reviewers collected the data from electronic and paper records. All identified charts were reviewed and assessed for eligibility. The case report forms were pilot-tested for the first 10 charts in duplicate to measure agreement and reproducibility among data collectors. Data dictionary and the case report forms were revised as necessary before completing the remainder of the cohort. Reviewers abstracted data for dialysis mode and dose, indication for dialysis, patient demographics, date of admission, ICU length of stay, comorbidities, baseline investigation including creatinine, lactate, hemoglobin, vasopressors use, thyroid and steroid replacement, mortality and CRRT-associated complications.
Agreement among the data collectors was assessed using the kappa statistic (crude kappa 0.80).

\section{Outcomes}

Primary outcomes included in-hospital and 30-day mortality. Secondary outcomes included ICU and hospital length of stay, reduction in serum lactate and creatinine levels, and the use of thyroid and glucocorticoid supplementation.

\section{Subgroup analysis}

Subgroup analysis was planned a priori examining the effects of CVVH compared with CVVHDF in postcardiac surgery patients admitted to the ICU, hypothesizing that the treatment effect will be larger in the CVVH group. The rationale for this was based on the authors' observation in clinical practice. The postcardiac surgery patients were more severely ill and required higher inotropic support. It was also unclear whether this subgroup would respond differently to higher flow during CRRT.

\section{Sample size}

Data collection was completed for 153 patients. The sample size was determined based on feasibility considerations. Using the rule of thumb of 10 to 15 events per predictor variable (22), a sample size of 153 with 86 events (mortality at 30 days) enabled the fitting of a logistic regression model with eight predictor variables.

\section{Statistical analysis}

Demographic data and medical conditions of the patients were analyzed to compare the difference between the patients in CVVH and CVVHDF group using descriptive statistics reported according to group as mean \pm SD for continuous variables and count (percentage) for categorical variables. P values from $\chi^{2}$ tests for categorical data and two-group $t$ tests for continuous data are reported. The two binary outcomes - hospital and 30-day mortality - were analyzed using logistic regression. The two continuous outcomes - ICU stay (in days) and hospital stay (in days) - were analyzed using linear regression. In the logistic and the linear regression, variables identified by comparing the group balance were adjusted for potential confounding factors. These variables included sex, ischemic heart disease, liver disease, hypertension, Acute Physiology and Chronic Health Evaluation (APACHE) score, central venous pressure and $24 \mathrm{~h}$ urine output before initiating CRRT. The results from linear regression are reported as adjusted difference between groups, corresponding two-sided 95\% CIs and associated $\mathrm{P}$ values. The same analyses were conducted for the subgroup of postcardiac surgery patients admitted to the ICU. Results from logistic regression are reported as OR $(95 \% \mathrm{CI})$ and associated P values. All statistical tests were performed using two-sided tests at the 0.05 level of significance; $\mathrm{P}$ values are reported to two decimal places. All analyses were performed using SAS version 9.2 (SAS Inc, USA).

\section{RESULTS}

A total of 178 charts were reviewed and 25 were excluded: 17 because of use of intermittent hemodialysis and eight because of missing information. Data were collected for 153 patients; of these, 59 received CVVHDF and 94 received CVVH for AKI in the ICU (Figure 1). The nursing educators maintained a log of any patients receiving CRRT, which enabled the identification of all potentially eligible patients. The dialysis flow rate was $35 \mathrm{~mL} / \mathrm{kg} / \mathrm{h}$ for all included patients. This flow rate was used before the publication of the Randomized Evaluation of Normal versus Augmented Level Replacement Therapy (RENAL) trial (23).

Given the lack of randomization and the heterogeneous sample, there were minor differences in baseline characteristics between the groups (Table 1). Although the APACHE II score was higher in the CVVH group relative to the CVVHDF group (29.18 versus 26.72; $\mathrm{P}=0.04$ ), the proportion of patients with comorbidities was higher in the CVVHDF group. The majority of patients underwent surgical intervention associated with ICU admission $(76 \%$ in the CVVH 
TABLE 1

\section{Baseline characteristics}

\begin{tabular}{|c|c|c|}
\hline Variable & CVVH (n=94) & CVVHDF $(n=59)$ \\
\hline Male sex & $51(54)$ & $47(80)$ \\
\hline Age, years & $67.28 \pm 15.30$ & $68.42 \pm 12.94$ \\
\hline Weight, kg & $88.14 \pm 24.12$ & $89.93 \pm 23.78$ \\
\hline Diabetes mellitus & $36(38)$ & $22(38)$ \\
\hline Ischemic heart disease & $50(53)$ & $41(71)$ \\
\hline Congestive heart failure & $18(19)$ & $12(21)$ \\
\hline Chronic lung disease & $23(25)$ & $10(17)$ \\
\hline Chronic renal insufficiency & $25(27)$ & $15(26)$ \\
\hline Liver disease & $2(2)$ & $8(14)$ \\
\hline Brain injury & $4(4)$ & $5(9)$ \\
\hline Hypertension & $53(56)$ & $16(28)$ \\
\hline Coronary artery bypass grafting & $13(14)$ & $10(17)$ \\
\hline Hypothyroidism & $8(18)$ & $12(20)$ \\
\hline Nonoperative & $22(24)$ & $16(29)$ \\
\hline \multicolumn{3}{|l|}{ RIFLE } \\
\hline Risk & $13(14)$ & $11(19)$ \\
\hline Injury & $19(20)$ & $18(31)$ \\
\hline Failure & $60(65)$ & $29(50)$ \\
\hline Loss & $1(1)$ & $0(0)$ \\
\hline End stage & $0(0)$ & $0(0)$ \\
\hline APACHE II score & $29.18 \pm 7.54$ & $26.72 \pm 6.45$ \\
\hline Central venous pressure, $\mathrm{mmHg}$ & $16.84 \pm 5.40$ & $18.80 \pm 4.98$ \\
\hline $24 \mathrm{~h}$ urine output, $\mathrm{mL}$ & $617.03 \pm 786.38$ & $930.44 \pm 1174.6$ \\
\hline Days & $3.79 \pm 2.75$ & $3.76 \pm 3.86$ \\
\hline
\end{tabular}

Data presented as mean $\pm S D$ or $n(\%)$. APACHE Acute Physiology and Chronic Health Evaluation; CABG Coronary artery bypass graft; $\mathrm{CVVH}$ Continuous venovenous hemofiltration; CVVHDF Continuous venovenous hemodiafiltration

group, $71 \%$ in CVVHDF group), and approximately 25\% in both groups had a history of chronic kidney disease that was not dialysis dependent. The indications for starting renal replacement therapy are summarized in Table 2. If more than one indication for dialysis was present, the one that was reported was considered to be the primary indication as described in the medical record.

Using univariate analysis, there was no difference in hospital or 30-day mortality between the groups. A trend toward shorter ICU length of stay was observed in the CVVH group (mean difference -27.51 [95\% CI -59.46 to 4.43]; $\mathrm{P}=0.09$ ) and a statistically significant reduction in hospital stay for CVVH (mean difference $-34.32[95 \%$ CI -68.03 to -0.60 ]; $\mathrm{P}=0.05$ (Table 3 ). After adjusting for multiple variables using multivariate regression analysis (Table 3 ), there was no difference in hospital mortality, 30-day mortality or ICU length of stay. However, there was a trend toward reduction in hospital length of stay in favour of the CVVH group (mean difference -34.14 [95\% CI -72.92 to 4.65 ]; $\mathrm{P}=0.08$ ).

Although serum lactate and creatinine levels for the first $48 \mathrm{~h}$ of CRRT were higher in the CVVH group, the difference was not statistically significant (Table 4). Interestingly, more patients required thyroid replacement when receiving CVVHDF (39\%) compared with those receiving CVVH (17\%) $(\mathrm{P}<0.01)$; the difference was statistically significant. Similarly, significantly more patients required glucocorticoids therapy in the CVVHDF (58\%) group compared with CVVH (36\%) $(\mathrm{P}<0.01)$, the main indication for glucocorticoids therapy was vasopressor refractory shock. The results are summarized in Table 4 .

\section{Subgroup analysis}

The postcardiac surgery population included 67 patients (40 in the CVVH group and 27 in the CVVHDF group). Subgroup analysis showed that hospital mortality was similar in both groups: 23 patients (57.7\%) died in the CVVH group compared with 15 (55.5\%) in the
TABLE 2

Indications for starting continuous renal replacement therapy

\begin{tabular}{lcc}
\hline Indication & CVVH ( $\mathbf{n = 9 4 )}$ & CVVHDF (n=59) \\
\hline AKI with reduced urine output & $70(74.47)$ & $41(69.49)$ \\
Acidosis* and AKI & $6(6.38)$ & $5(8.47)$ \\
Abdominal compartment syndrome & $2(2.13)$ & $0(0.00)$ \\
Salicylate toxicity & $1(1.06)$ & $0(0.00)$ \\
Pulmonary odema & $6(6.38)$ & $9(15.25)$ \\
Thrombotic thrombocytopenic purpura & $1(1.06)$ & $0(0.00)$ \\
Oliguria $^{\dagger}$ & $4(4.26)$ & $0(0.00)$ \\
Rhabdomyolysis $^{\ddagger}$ with AKI & $1(1.06)$ & $0(0.00)$ \\
Uremia & $3(3.19)$ & $3(5.08)$ \\
Not clear & $0(0.00)$ & $1(1.69)$ \\
\hline
\end{tabular}

Data presented as $n$ (\%). ${ }^{*} p h<7.2$ with acute kidney injury (AKI); ${ }^{+}$Oliguria Urine output $<500 \mathrm{~mL} / 24 \mathrm{~h}$; 抽evated creatine kinase level and associated AKI not responsive to fluid management. CVVH Continuous venovenous hemofiltration; CVVHDF Continuous venovenous hemodiafiltration

CVVHDF group (OR 1.08 [95\% CI 0.4 to 2.90]; $\mathrm{P}=0.87$ ). Mortality at 30 days, ICU and hospital length of stay were similar in both groups (Table 5). Multiple regression analysis adjusting for variables such as sex, pre-existing ischemic heart disease and APACHE II score yielded similar results (Table 5).

\section{DISCUSSION}

In the present retrospective study, we did not find any differences in hospital or 30-day mortality when comparing CVVH with CVVHDF. The present study included a wide spectrum of critically ill patients admitted to the neurotrauma, medical and cardiac surgery ICUs. Baseline APACHE II scores were high, which reflect a high predicted mortality at baseline (Table 1). Hence, hospital mortality was high but consistent with the recent literature (1). Fifty-eight percent of patients died in the CVVH group compared with 64\% in the CVVHDF group and, although the results were numerically different, multivariate logistic regression analysis did not yield a statistically significant difference. ICU length of stay was similar in both groups; however, there was a trend toward reduction in hospital length of stay in the CVVH group. Although the difference could be due to chance, we cannot rule out other factors that could explain this observation.

Some studies have suggested that CRRT increases clearance of beneficial hormones such as thyroid-stimulating hormone, parathyroid hormone, cortisol, aldosterone, insulin and, possibly, testosterone $(24,25)$. The clinical implication of this observation is unknown. In our study, we did not measure the clearance of any hormones but we described the proportion of patients who received thyroid replacement and glucocorticoids; the use of both hormones was higher in the CVVHDF group and the difference was statistically significant $(\mathrm{P}=0.01)$ (Table 4). It is possible that the temporal nature of the interventions influenced the observed results. CVVHDF was used in our institution before 2008, in which glucocorticoids were also more frequently prescribed to critically ill patients with septic shock than over the past few years. We cannot draw any firm conclusions based on these observations, but it may generate hypotheses to be validated in future studies. Moreover, we observed lower serum lactate and creatinine levels with CVVH at $48 \mathrm{~h}$ but the difference was not statistically significant (Table 4). Although a firm conclusion cannot be drawn, this may be an indirect measure of the efficiency of CVVH.

We hypothesized that postcardiac surgery patients will have more benefit with convective therapy using CVVH because these patients carry high risk for death in the context of AKI requiring dialysis postoperatively (21). However, subgroup analysis did not reveal any difference between the groups in all clinical and biochemical outcomes.

Over the past few years, CVVH has become widely used in ICU settings (20). This is possibly related to the theoretical advantage of 
TABLE 3

Primary outcomes: Univariate and multivariate regression analysis

\begin{tabular}{|c|c|c|c|c|}
\hline Outcome & CVVH & CVVHDF & Univariate analysis & Multivariate analysis* \\
\hline Mortality at 30 days, $\mathrm{n} / \mathrm{n}(\%)$ & $54 / 94(57)$ & $32 / 59(54)$ & $\mathrm{OR}^{\dagger} 1.14(95 \% \mathrm{Cl} 0.59$ to 2.19$) ; \mathrm{P}=0.70$ & OR $1.35(95 \% \mathrm{Cl} 0.62$ to 2.95$) ; \mathrm{P}=0.45$ \\
\hline Hospital mortality, n/n (\%) & $55 / 94(59)$ & $37 / 58(64)$ & OR $0.80(95 \% \mathrm{Cl} 0.41$ to 1.57$) ; \mathrm{P}=0.52$ & OR $0.85(95 \% \mathrm{Cl} 0.38$ to 1.89$) ; \mathrm{P}=0.69$ \\
\hline ICU length of stay, days, mean & 19.35 & 46.84 & $\mathrm{MD}^{\ddagger}-27.5(95 \% \mathrm{Cl}-59.46$ to 4.43$) ; \mathrm{P}=0.09$ & $\mathrm{MD}-28.22(95 \% \mathrm{Cl}-65.26$ to 8.81$) ; \mathrm{P}=0.14$ \\
\hline Hospital stay, days, mean & 28.53 & 62.85 & $\mathrm{MD}-34.32(95 \% \mathrm{Cl}-68.03$ to -0.60$) ; \mathrm{P}=0.05$ & $\mathrm{MD}-34.14(95 \% \mathrm{Cl}-72.92$ to 4.65$) ; \mathrm{P}=0.08$ \\
\hline
\end{tabular}

${ }^{*}$ All models were adjusted for sex, ischemic heart disease, liver disease, hypertension, Acute Physiology and Chronic Health Evaluation score, pre-central venous pressure, 24 h urine output and pre-continuous renal replacement therapy, which were identified by comparing the group balance. Backward stepwise regressions were used for variable; †OR from logistic model; ${ }^{\ddagger} M D$ Mean difference from linear regression model. CVVH Continuous venovenous hemofiltration; CVVHDF Continuous venovenous hemodiafiltration; ICU Intensive care unit

\section{TABLE 4}

\section{Secondary outcomes}

\begin{tabular}{lccr}
\hline Outcome & CVVH (n=94) & CVVHDF (n=59) & P $^{*}$ \\
\hline Glucocorticoids, $\mathrm{n}(\%)$ & $34(36)$ & $34(58)$ & $<0.01$ \\
Lactate clearance, mmol/L, mean \pm SD & & \\
Day 1 & $-0.86 \pm 5.52$ & $-1.43 \pm 4.23$ & 0.52 \\
Day 2 & $-2.19 \pm 6.09$ & $-1.24 \pm 4.04$ & 0.33 \\
Thyroid replacement, n (\%) & $16(17)$ & $23(39)$ & $<0.01$ \\
\hline
\end{tabular}

${ }^{*} \chi^{2}$ test for categorical data and two-group $\mathrm{t}$ test for continuous data. CVVH Continuous venovenous hemofiltration; CVVHDF Continuous venovenous hemodiafiltration

increased clearance of higher molecular weight molecules and inflammatory cytokines, especially in septic patients $(14,26,27)$. There are no large randomized controlled trials comparing the two modalities. Recently, a multicentre Canadian pilot study (OMAKI [28]) compared the two modalities in critically ill patients and showed that mortality (54\% CVVH; 55\% CVVHD) and dialysis dependence in survivors (24\% CVVH; 19\% CVVHD) at 60 days were similar. However, a larger randomized controlled trial is needed in this area.

The strengths of our review include using standardized case report forms for data abstraction; the use of robust statistical methods to test our hypothesis; and examining the effect of interventions on important patient outcomes and laboratory markers. However, a few limitations are worth mentioning. Our study was perhaps underpowered to detect a mortality difference (if a difference truly exists); this is mainly related to the small sample size. Similar to other retrospective cohort studies, our review was subject to biases, especially in outcome assessment because it is challenging to acquire verifiable data based on retrospective data collection. Patient selection (selection bias) may play a role in choosing the dialysis mode in which sicker patients received CVVH; this is suggested by the difference in baseline characteristics in both groups (Table 1). Data regarding delivered dose of dialysis were not collected, although high doses $(35 \mathrm{~mL} / \mathrm{kg} / \mathrm{h})$ were prescribed. It is challenging to ascertain whether the delivered dose was similar to the prescribed dose. Data regarding eight patients were missing and they were not included in the analysis (Figure 1). Although we used multivariable analysis adjusting for different confounding variables, it is challenging and sometimes impossible to control for all confounding variables in a nonrandomized study design.

\section{CONCLUSION}

The present retrospective review suggests that the use of CVVH is not associated with improved survival when compared with CVVHDF. Although the use of CVVH was associated with shorter hospital stay, the difference was not statistically significant. A large randomized controlled trial is warranted before we can draw any firm conclusions. Future research should examine the clinical consequences of hormonal and other biomarkers clearance.

\section{TABLE 5}

Univariate and multivariate analyses for cardiovascular intensive care unit (ICU) patient outcomes

\begin{tabular}{|c|c|c|c|c|}
\hline Outcome & CVVH & CVVHDF & Univariate analysis & Multivariate analysis* \\
\hline Mortality at 30 days, n/n (\%) & $23 / 40(57)$ & $15 / 27(55)$ & $\mathrm{OR}^{\dagger} 1.08(95 \% \mathrm{Cl} 0.40$ to 2.90$) ; \mathrm{P}=0.87$ & OR $0.90(95 \% \mathrm{Cl} 0.28$ to 2.88$) ; \mathrm{P}=0.86$ \\
\hline Hospital mortality, n/n (\%) & $23 / 40(58)$ & $17 / 27(63)$ & OR $0.72(95 \% \mathrm{Cl} 0.26$ to 1.99$) ; \mathrm{P}=0.52$ & OR $0.53(95 \% \mathrm{Cl} 0.16$ to 1.78$) ; P=0.30$ \\
\hline ICU length of stay, days, mean & 17.8 & 40.48 & $\mathrm{MD}^{\ddagger}-2.66(95 \% \mathrm{Cl}-10.91$ to 5.60$) ; \mathrm{P}=0.53$ & $\mathrm{MD}-4.87(95 \% \mathrm{Cl}-13.94$ to 4.20$) ; \mathrm{P}=0.29$ \\
\hline Hospital stay, days, mean & 28.2 & 30.7 & $\mathrm{MD}-2.52(95 \% \mathrm{Cl}-14.46$ to 9.43$) ; \mathrm{P}=0.68$ & $\mathrm{MD}-4.13(95 \% \mathrm{Cl}-17.73$ to 9.46$) ; \mathrm{P}=0.55$ \\
\hline
\end{tabular}

${ }^{*}$ All models were adjusted for sex, ischemic heart disease, liver disease, hypertension, Acute Physiology and Chronic Health Evaluation score, pre-central venous pressure, $24 \mathrm{~h}$ urine output and pre-continuous renal replacement therapy, which were identified by comparing the group balance. Backward stepwise regressions were used for variable; tOR from logistic model; ${ }^{\ddagger} M D$ Mean difference from linear regression model. CVVH Continuous venovenous hemofiltration; CVVHDF Continuous venovenous hemodiafiltration

\section{REFERENCES}

1. Uchino S, Kellum JA, Bellomo R, et al. Acute renal failure in critically ill patients: A multinational, multicenter study. JAMA 2005;294:813-8.

2. Mehta RL, McDonald B, Gabbai FB, et al. A randomized clinical trial of continuous versus intermittent dialysis for acute renal failure. Kidney Int 2001;60:1154-63.

3. Augustine JJ, Sandy D, Seifert TH, Paganini EP. A randomized controlled trial comparing intermittent with continuous dialysis in patients with ARF. Am J Kidney Dis 2004:44:1000-7.

4. Uehlinger DE, Jakob SM, Ferrari P, et al. Comparison of continuous and intermittent renal replacement therapy for acute renal failure. Nephrol Dialysis Transpl 2005;20:1630-7.

5. Vinsonneau C, Camus C, Combes A, et al. Continuous venovenous haemodiafiltration versus intermittent haemodialysis for acute renal failure in patients with multiple-organ dysfunction syndrome: A multicentre randomised trial. Lancet 2006;368:379-85.
6. Bagshaw SM, Berthiaume LR, Delaney A, Bellomo R. Continuous versus intermittent renal replacement therapy for critically ill patients with acute kidney injury: A meta-analysis. Crit Care Med 2008;36:610-7.

7. Klouche K, Cavadore P, Portales P, Clot J, Canaud B, Beraud JJ. Continuous veno-venous hemofiltration improves hemodynamics in septic shock with acute renal failure without modifying TNFalpha and IL6 plasma concentrations. J Nephrol 2002;15:150-7.

8. Heering P, Morgera S, Schmitz FJ, et al. Cytokine removal and cardiovascular hemodynamics in septic patients with continuous venovenous hemofiltration. Intens Care Med 1997;23:288-96.

9. Bellomo R, Tipping P, Boyce N. Interleukin-6 and interleukin-8 extraction during continuous venovenous hemodiafiltration in septic acute renal failure. Renal Fail 1995;17:457-66.

10. Sanchez-Izquierdo JA, Perez Vela JL, Lozano Quintana MJ, Alted Lopez E, Ortuno de Solo B, Ambros Checa A. Cytokines 
clearance during venovenous hemofiltration in the trauma patient. Am J Kidney Dis 1997;30:483-8.

11. Ronco C, Tetta C, Mariano F, et al. Interpreting the mechanisms of continuous renal replacement therapy in sepsis: The peak concentration hypothesis. Artific Organs 2003;27:792-801.

12. Silvester W. Mediator removal with CRRT: Complement and cytokines. Am J Kidney Dis 1997;30:S38-43.

13. Bellomo R, Tipping P, Boyce N. Continuous veno-venous hemofiltration with dialysis removes cytokines from the circulation of septic patients. Critical Care Med 1993;21:522-6.

14. van Bommel EF, Hesse CJ, Jutte NH, Zietse R, Bruining HA, Weimar W. Impact of continuous hemofiltration on cytokines and cytokine inhibitors in oliguric patients suffering from systemic inflammatory response syndrome. Renal Fail 1997;19:443-54.

15. Brunet S, Leblanc M, Geadah D, Parent D, Courteau S, Cardinal J. Diffusive and convective solute clearances during continuous renal replacement therapy at various dialysate and ultrafiltration flow rates. Am J Kidney Dis 1999;34:486-92.

16. Troyanov S, Cardinal J, Geadah D, et al. Solute clearances during continuous venovenous haemofiltration at various ultrafiltration flow rates using Multiflow-100 and HF1000 filters. Nephrol Dialysis Transpl 2003;18:961-6.

17. Gatward JJ, Gibbon GJ, Wrathall G, Padkin A. Renal replacement therapy for acute renal failure: A survey of practice in adult intensive care units in the United Kingdom. Anaesthesia 2008;63:959-66.

18. Langford S, Slivar S, Tucker SM, Bourbonnais FF. Exploring CRRT practices in ICU: A survey of Canadian hospitals. Dynamics 2008;19:18-23.

19. Renal replacement therapy for acute kidney injury in Australian and New Zealand intensive care units: A practice survey. Crit Care Resuscitat 2008;10:225-30.

20. Uchino S, Bellomo R, Morimatsu H, et al. Continuous renal replacement therapy: A worldwide practice survey. The beginning and ending supportive therapy for the kidney (B.E.S.T. kidney) investigators. Intens Care Med 2007;33:1563-70.

21. Luckraz H, Gravenor MB, George R, et al. Long and short-term outcomes in patients requiring continuous renal replacement therapy post cardiopulmonary bypass. Eur J Cardiothorac Surg 2005;27:906-9.

22. Peduzzi P, Concato J, Kemper E, Holford TR, Feinstein AR. A simulation study of the number of events per variable in logistic regression analysis. J Clin Epidemiol 1996;49:1373-9.

23. Bellomo R, Cass A, Cole L, et al. Intensity of continuous renalreplacement therapy in critically ill patients. N Engl J Med 2009;361:1627-38.

24. Bellomo R, Colman PG, Caudwell J, Boyce N. Acute continuous hemofiltration with dialysis: Effect on insulin concentrations and glycemic control in critically ill patients. Crit Care Med 1992;20:1672-6.

25. Bellomo R, McGrath B, Boyce N. Effect of continuous venovenous hemofiltration with dialysis on hormone and catecholamine clearance in critically ill patients with acute renal failure. Crit Care Med 1994;22:833-7.

26. Hoffmann JN, Hartl WH, Deppisch R, Faist E, Jochum M, Inthorn D. Hemofiltration in human sepsis: Evidence for elimination of immunomodulatory substances. Kidney Int 1995;48:1563-70.

27. Kellum JA, Johnson JP, Kramer D, Palevsky P, Brady JJ, Pinsky MR. Diffusive vs. convective therapy: Effects on mediators of inflammation in patient with severe systemic inflammatory response syndrome. Crit Care Med 1998;26:1995-2000.

28. Wald R, Friedrich JO, Bagshaw SM, et al. Optimal Mode of clearance in critically ill patients with Acute Kidney Injury (OMAKI) - a pilot randomized controlled trial of hemofiltration versus hemodialysis: A Canadian Critical Care Trials Group project. Crit Care 2012;16:R205. 


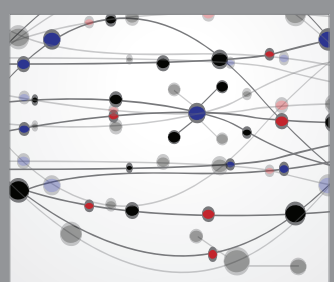

The Scientific World Journal
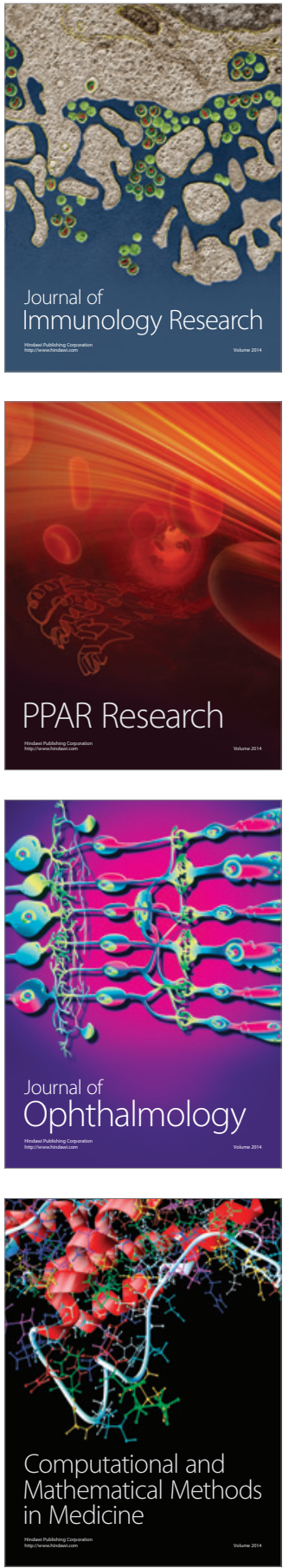

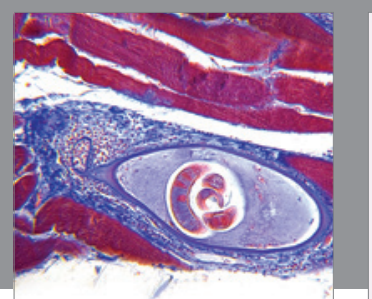

Gastroenterology Research and Practice

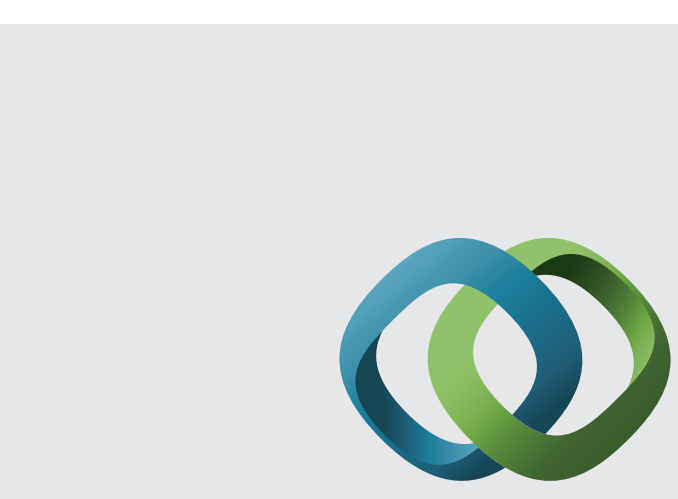

\section{Hindawi}

Submit your manuscripts at

http://www.hindawi.com
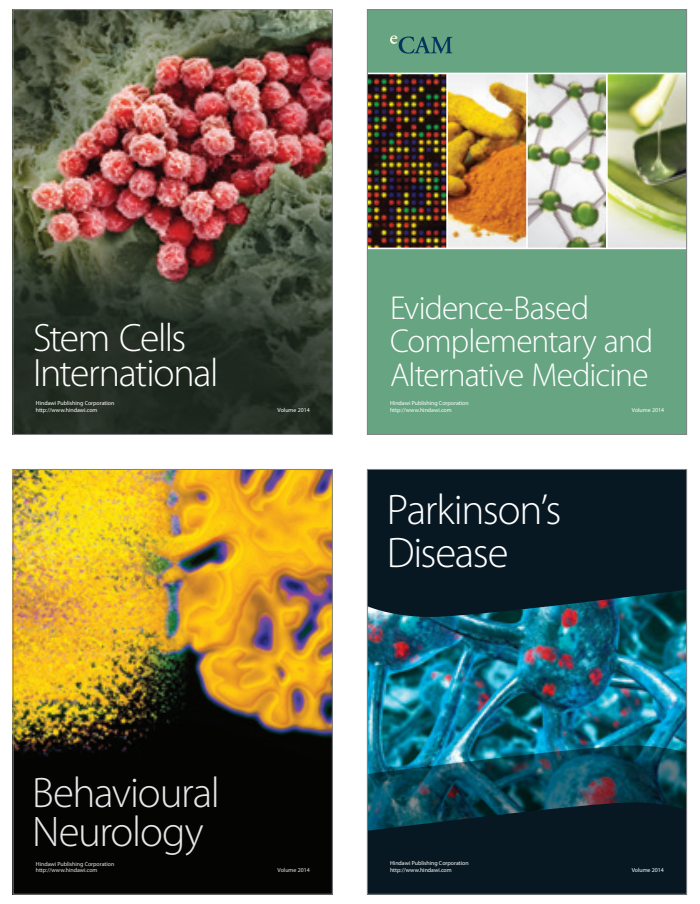
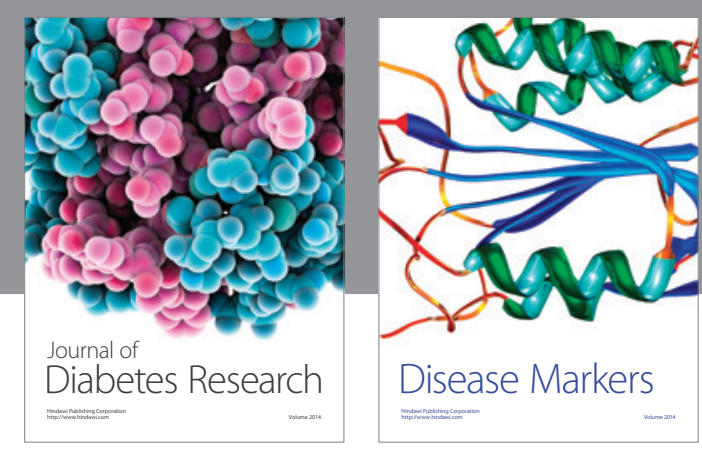

Disease Markers
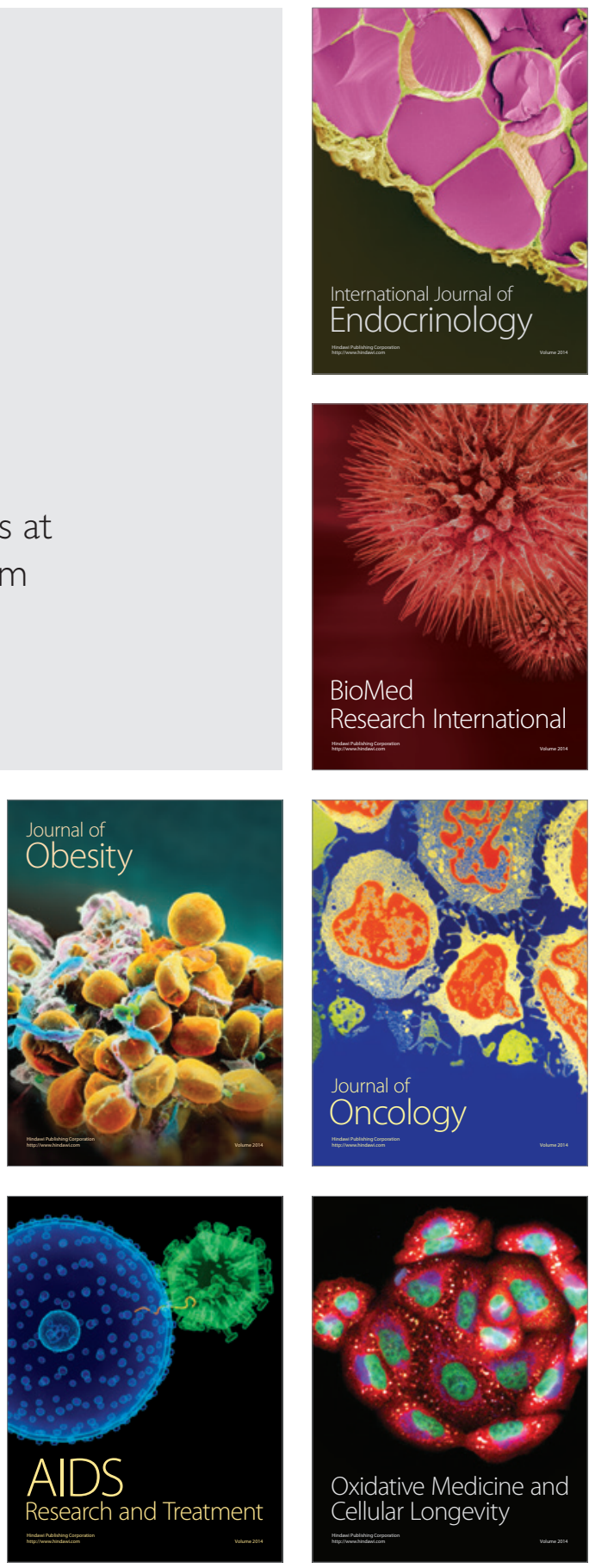